\title{
Entre Caminos y Equinos: Un Modelo SIG para Localizar Sitios Arqueológicos de Momentos Históricos Ecuestres (Provincia de Santa Cruz, Argentina).
}

\author{
Between Path and Horses: A Gis Model to Locate Archaeological Sites of Equestrian \\ Historical Times (Province of Santa Cruz, Argentina).
}

Natalia Andrea Cirigliano y María Cecilia Palloi

\section{RESUMEN}

Los modelos de rutas óptimas son herramientas útiles dentro de los Sistemas de Información Geográfica (SIG) que permiten estimar zonas propicias para la circulación de humanos y otras especies de animales. En particular para la circulación ecuestre, estos modelos generan expectativas sobre redes de caminos que pueden ser contrastadas con la información arqueológica y las fuentes escritas sobre espacios ocupados por grupos indígenas ecuestres de tiempos históricos. Aquí se presenta un análisis espacial con datos geográficos, etnohistóricos e históricos de los grupos ecuestres que ocuparon el área comprendida entre las cuencas de los ríos Chico y Santa Cruz (Provincia de Santa Cruz, Argentina). Dado que los sitios arqueológicos de tiempos históricos detectados hasta el momento son escasos, adquiere cada vez más importancia evaluar el potencial del área para reconocerlos. Los resultados del modelo nos dan pistas sobre cómo reorientar la búsqueda arqueológica hacia espacios que aún no han sido prospectados. Adicionalmente, generan expectativas sobre la evidencia esperable para distintos tipos de asentamiento, incluyendo ocupaciones estacionales o de más largo plazo.

Palabras Clave: Modelo De Rutas Óptimas, SIG, Cazadores Recolectores Ecuestres, Patagonia Meridional.

\section{ABSTRACT}

Least cost path models are useful tools within GIS that allow to estimate conducive areas to the movement of humans and other species of animals. Particularly for the equestrian mobility, these models generate expectations on path networks that can be tested against archaeological information and written sources on spaces occupied by indigenous equestrian groups of historic times. Here, a spatial analysis with geographic, ethnohistorical and historical data of equestrian groups that occupied the area between Chico and Santa Cruz river basins (Province of Santa Cruz, Argentina) is presented. Due to archaeological sites of historical times detected so far are scarce, assessing the potential of the area to recognize them is becoming increasingly important. Model results give us clues about how the archaeological search could be re-focused to spaces that have not yet been prospected. Additionally, they

i CONICET-IMHICIHU. Saavedra 15, $5^{\circ}$ piso, Argentina. Correo-e: naticirigliano@hotmail.com.

ii CONICET-IMHICIHU. Saavedra 15, 5 piso, Argentina. Correo-e: ceciliapallo@gmail.com 
raise expectations about the expected evidence for different types of settlement, including seasonal or longer term occupations.

Key Words: Optimal Path Model, Gis, Equestrian Hunter-Gatherers, Southern Patagonia.

\section{INTRODUCCIÓN}

La región entre los ríos Chico y Santa Cruz ha sido objeto de distintos trabajos arqueológicos focalizados en analizar la organización tecnológica, las formas de circulación humana y el efecto de la disponibilidad diferencial de recursos sobre la ocupación humana durante los últimos 11000 años (e.g. Borrero y Carballo Marina 1998; Carballo Marina et al. 1999; Franco 2002, 2008; Franco et al. 2007a y b, Franco et al. 2010, Franco et al. 2013a y b). En función de ello se han explorado sectores con características ambientales diferentes (Figura I).

Hacia el oeste del área, las grandes cuencas pericordilleranas de los lagos Argentino y Viedma están ubicadas en un paisaje glaciar, rodeadas de montañas con alturas máximas de 1500 msnm (Furque 1973). En la porción central y hasta alcanzar la costa atlántica hacia el este, el paisaje se constituye principalmente de mesetas sedimentarias y basálticas -incluyendo niveles aterrazados-, disectadas por valles fluviales y cañadones, cuyas alturas varían entre 1200 y 200 msnm (Panza et al. 2005). El clima es fríoárido de meseta central y costero, con promedios de $10^{\circ}$ a $8^{\circ} \mathrm{C}$. La precipitación anual decrece en sentido oeste-este con un máximo invernal, desde los 400 $\mathrm{mm}$ anuales en las zonas pericordilleranas hasta los 150 a $200 \mathrm{~mm}$ anuales entre la meseta central y la costa (Oliva et al. 200I; Soto 2000). En particular, la meseta central queda fuera del área de influencia de los lagos cordilleranos y la costera atlántica, sufriendo una gran amplitud en su temperatura media anual y una intensa evaporación. Esto se traduce en que buena parte del sector estudiado presenta actualmente veranos cálidos e inviernos muy fríos, con vientos particularmente intensos de primavera-verano (Oliva et al. 200 I). En el pasado el clima ha sido oscilante, principalmente en términos de períodos más húmedos y más áridos (Mancini et al. 2012; Stine 1994; entre otros).

La presencia humana en el área se registra desde la transición Pleistoceno-Holoceno hasta tiempos recientes (Franco et al. 2007a y b, Franco et al. 20l0, Franco et al. 20l3a y b, entre otros). En particular para el período histórico, las fuentes escritas señalan que los primeros contactos hispanoindígenas se establecieron en la costa hacia I520 DC, mientras que las primeras incursiones alcanzaron escasos kilómetros hacia el interior (e.g. Narborough 2007 [ I694]; Pigaffeta 200 I [ 1899]). Para el siglo XVIII

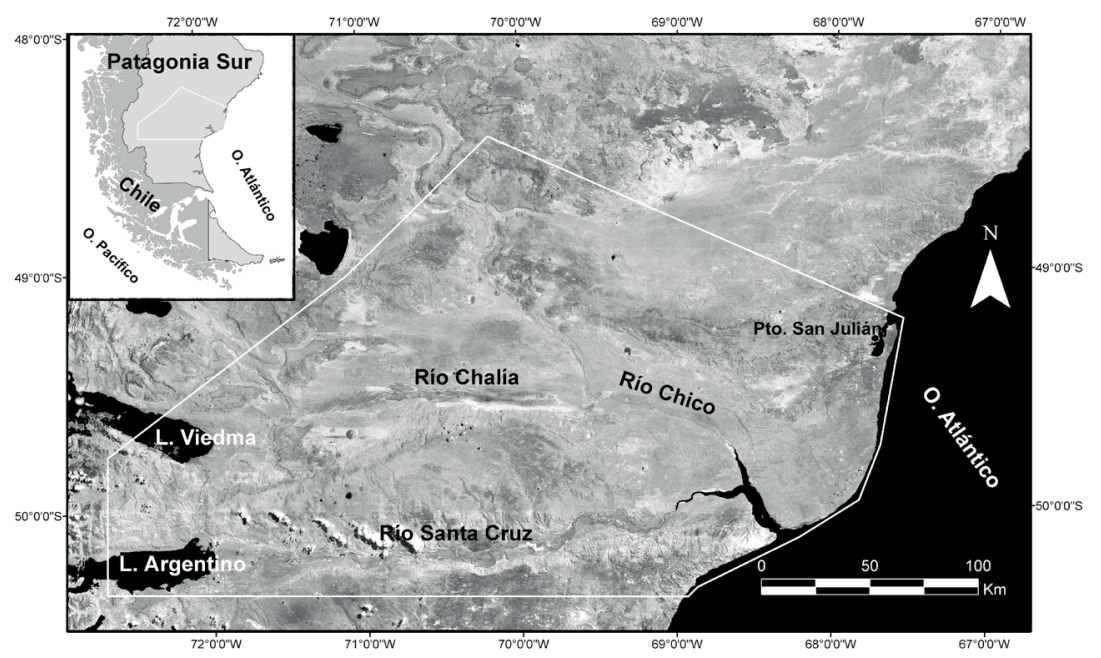

Figura 1: Área de estudio.

Figure 1: Study area. 
los documentos mencionan la presencia y circulación de grupos cazadores-recolectores ecuestres (e.g. Viedma 1972 [1780-1783]). Dicha información se vuelve más numerosa para los siglo $\mathrm{XIX}$ y $\mathrm{XX}$ (e.g. Burmeister 1892 [189I]; Musters 2005 [191I]; Moreno 2007 [1877]), mientras que durante los siglos $X X$ y $X X I$ se suman algunos relatos recopilados a partir de la tradición oral (e.g. Gradin 2000; Halvorsen 20I I; Priegue 2007).

A pesar de la rica información provista por las fuentes orales y escritas, las distintas investigaciones arqueológicas han detectado escasos sitios arqueológicos asignables a momentos de contacto y poscontacto tanto en el área de estudio (Cirigliano y Vommaro 20I3, 20I4) como en zonas próximas (e.g. Buscaglia 2012; Goñi 20I3). Dada la baja frecuencia de sitios para el período ca. 1750 DC y la primera mitad del siglo XX, proponemos generar un modelo sobre rutas óptimas que contribuya a localizarlos. Este modelo se desarrolla en un entorno SIG y contempla la geografía del área, la disponibilidad potencial de los espacios para su uso estacional o de más largo plazo, y las características del sistema de movilidad ecuestre junto con la localización de los sitios históricos mencionados en las fuentes escritas.

\section{MOVILIDADY USO DEL ESPACIO DE LOS CAZADORES RECOLECTORES ECUESTRES ENTRE LOS RÍOS CHICOY SANTA CRUZ}

La incorporación del caballo como tecnología de transporte alteró la movilidad de los grupos indígenas aumentando los rango de acción, reduciendo los costos del traslado, favoreciendo el transporte de objetos y la búsqueda de determinados recursos (e.g. Binford 200 I; Boschín y Nacuzzi 1979; Goñi 2000, 20I3; Kelekna 2009; Kelly 1995; Martinic 1984, 1995; Matteucci y Scheinsohn 2004). Según algunos investigadores, el caballo habría intensificado el modo de vida nómade (e.g. Boschín y Nacuzzi 1979; Kelly 1995;Wissler 1914;), generando la dependencia de sectores bajos con aguas y pasturas (e.g. Ewers 1955; Goñi 2000, 20 I 3; Matteucci y Scheinsohn 2004; Moreno 2008; Zedeño et al. 20l4).

En relación con algunas de las rutas indígenas en Patagonia, Nacuzzi y Pérez de Micou (1994) plantean que seguían los cursos de los cañadones, las lagunas y las aguadas. Para el área entre los ríos Chico y Santa Cruz, esto mismo se expresa en las crónicas de viajeros (e.g. Musters 2005 [ I9II], Viedma 1972 [1780-1783]) y en los trabajos de investigadores (e.g. Rey Balmaceda 1960; Boschín y Nacuzzi 1979). Otros estudios enfatizan de manera particular el uso de cañadas o cañadones que desembocan en la margen norte del río Santa Cruz (e.g. Fernández Garay 1994; Gradin 2000) y la importancia de zonas sin rocas, ya que éstas perjudican los cascos de los caballos (Moyano 188I). En su viaje desde el río Santa Cruz hasta la colonia de Chubut Moyano relata:

"Conozco el modo de ser de los tehuelches y sabía bien que ellos no van ni pasan casi nunca sino por parages donde pueden irse deteniendo varios días para hacer las cacerías de que exclusivamente viven, trazando sus sendas donde no hay piedras que destruyan sus caballos sin herradura en las marchas $y$ en las boleadas, y además, como tienen pocos caballos; su toldo y su ajuar lo trasportan en solo dos ó tres cargas muy pesadas, con las cuales solo pueden andar cinco o seis leguas por día, teniendo por consiguiente, que detenerse en sitios con suficiente agua y pasto para las yeguadas de toda la tribu" (Moyano I88I:5).

Por su parte, los lugares elegidos como paraderos durante momentos ecuestres presentaban agua, pastos, leña y abrigos para resguardarse del viento (Nacuzzi 2005). Los mismos eran designados con la palabra ayke entre los tehuelches -que significa alojamiento, parada o lugar- y reunían los cuatro elementos principales - carne, agua, pasto y leña(del Castillo 1979 [1887]: 35). De acuerdo con el tiempo de permanencia del grupo y el número de toldos en un paraje, para el lapso I770- I870 existían distintos tipos de asentamientos tehuelches en Patagonia meridional. Nacuzzi (199I, 2005) describe cuatro tipos que denomina: I- campamentos base; 2- asentamientos próximos en áreas de aprovisionamiento; 3- asentamientos transitorios durante traslados; y 4- grandes asentamientos múltiples (Nacuzzi I99I, 2005). Entre ellos, Boschín y Nacuzzi (1979) advierten que la mayoría de los lugares para acampar en Patagonia meridional eran postas durante los traslados, en las que los indígenas pasaban tres días aproximadamente (e.g. Musters 2005 [191 I];Viedma 1972 [1780-I783]). 
Las crónicas de viajeros mencionan el paso por diversos parajes en cañadas con agua y pasturas, y en algunos casos con el aditivo de leña y caza. Un ejemplo de ello corresponde al recorrido que Viedma hizo junto a una partida de indígenas desde Bahía San Julián hasta el lago Capar (Viedma) en dirección este - oeste y luego oeste - este. El 3 de abril de 1780 Viedma señaló lo siguiente sobre el campamento indígena en Bahía San Julián:

"A media legua de la playa entramos en una cañada bien ancha. $A$ un cuarto de legua más llegamos a la toldería de los indios... A la orilla de la toldería había cinco pozos de agua dulce, que estaba muy sucia de los caballos, seguimos la cañada arriba... encontramos con el manantial que brota por dos partes... subí luego a una inmediata loma, y en cuanto alcancé con la vista, advertí todo el terreno vestido de pastos, formando iguales lomas y cañadas; pero en estas, se reconocía muy alta y espesa la yerba; y en punta a leña, se veía muy poca y menuda, desde que se aleja de la playa como media legua. En todas estas cañadas pueden proporcionarse con el tiempo muy buenas huertas y árboles..." (Viedma 1972 [l 780-I 783]: 66-67).

También los asentamientos y/o la presencia de europeos o criollo europeos en el territorio pudieron haber funcionado como atractores para los grupos indígenas (e.g. Musters 2005 [1911]; Viedma (1972 [1780-I783], aunque también ver Aguerre 2000). Lugares como Floridablanca, Carmen de Patagones, Isla Pavón, Punta Arenas y Cañadón León (actualmente Gobernador Gregores) sirvieron para el intercambio y el comercio (e.g.Aguerre 2000; Musters 2005 [1911]); Viedma (1972 [1780-1783]), por lo que su localización podría haber alterado los circuitos de movilidad ecuestre. Cabe señalar que en este contexto se produjo el avance de la frontera ovino ganadera. Esto último a partir de 1880 DC en la provincia de Santa Cruz, y específicamente para el área de estudio a partir del siglo $\mathrm{XX}$, incluyendo la creación de reservas indígenas (Barbería 1995).

De acuerdo con estudios sobre la disponibilidad de los recursos para los animales, es posible que durante el invierno escasearan las pasturas para el forrajeo a causa de la nieve y sólo algunos lugares fueran aptos para su uso (Osborn 1983;
Raedeke 1979). En relación con esto, la información aportada por las fuentes da cuenta de lugares de uso estacional, por ejemplo el lago Capar (lago Viedma). En cercanías de ese lago el paraje conocido como Ayr fue visitado por una partida de indígenas, quienes junto con Viedma arribaron allí el 21 de noviembre de 1782 (Viedma 1972 [I780-I783]: 66-67). Según Patricio -hermano del cacique Julián- debían irse del paraje porque el derretimiento de la nieve -a causa de los vientos y el sol- haría imposible vadear los arroyos para regresar $y$ tendrían que pasar el verano allí hasta el comienzo de las heladas. En cambio, el paradero Frequent Winter Quarters of Tehuelches (Musters 2005 [19II]) -localizado en cercanías de la confluencia de los ríos Chico y Chalía- podría haber sido un asentamiento fijo durante la estación invernal (Nacuzzi 1991, 2005). También Molinari ofrece información de los movimientos estacionales durante los años 1903 y 1904.

“...Hay también algunos indios, pero éstos no tienen sino toldos que en la temporada de octubre a marzo los cambian continuamente en los cañadones de la alta meseta al Sur del Chalía, bajando en abril al valle de dicho arroyo y río Chico para pasar el invierno." (Molinari en Halvorsen 2009:27).

En conjunto, los documentos históricos sobre el uso de caballos entre los grupos indígenas remarcan las ocupaciones en espacios bajos, amplios, abiertos y reparados de los vientos predominantes, mientras que zonas con afloramientos rocosos o con insuficiente disponibilidad de agua y pasturas eran evitadas (Boschín y Nacuzzi 1979; del Castillo 1979; Goñi 20I3; Matteucci y Scheinsohn 2004; Musters 2005 [ 191 I]; Nacuzzi 2005; Nacuzzi y Pérez de Micou 1994; Moyano I88I; Viedma 1972 [I780-I783]:6667). La estacionalidad también parece haber jugado un papel clave, actuando como condicionante en la selección de lugares para ocupar (e.g. Musters 2005 [19II]; Nacuzzi 199I, 2005; Viedma 1972 [17801783]). De acuerdo con el corpus de información presentado es esperable una mayor frecuencia de sitios de paso o transitorios en el área de estudio (Boschín y Nacuzzi 1979; Cirigliano 2016), y por lo tanto el predominio de bajas frecuencias de hallazgos arqueológicos (Borrero et al. 2008). Aún considerando que los sitios de paso o transitorios podrían tener una baja visibilidad arqueológica, entendemos que es posible que el uso recurrente 
de estos lugares quede registrado en el patrón promediado de los contextos arqueológicos en una escala regional (Binford 1980). Dada la dificultad que encierra su detección (Cirigliano 2016), creemos que un punto de partida sumamente útil para abordar su búsqueda deriva de reconocer potenciales vías de circulación ecuestre, que también pueden servir para identificar otros tipos de asentamientos (sensu Nacuzzi 1991, 2005).

\section{METODOLOGÍA}

Los requerimientos de la movilidad de los grupos indígenas ecuestres sirvieron de base para seleccionar los aspectos físicos del terreno que permiten predecir rutas óptimas. De acuerdo con esto, se consideraron I- la pendiente y 2- la altura del terreno, principalmente vinculadas con las condiciones del traslado y el descanso de los caballos; 3 - los tipos de comunidades vegetales y su valor de productividad primaria, dada la importancia de la disponibilidad de abundante forraje -gramíneaspara los animales; 4- las fuentes y cursos de agua, que son un recurso crítico en Patagonia tanto para humanos como para los animales (e.g. Borrero 2005; Borrero y Franco 2000; Goñi 20 I3); 5 - la presencia de afloramientos rocosos como espacios no aptos para el galope del caballo (e.g. Goñi 20I3; Moyano I88I); y 6- la caída de nieve, vinculada con la disponibilidad de espacios para ser ocupados y transitados durante el invierno (Osborn 1983; Zedeño et al. 2014).

Para modelar las rutas óptimas se empleó el software ESRI ArcMap 9.3 y el modelo digital de elevación del proyecto ASTER Global Digital Elevation Model (ASTER GDEM), del que se reclasificaron la elevación y la pendiente del terreno. La elevación se consideró en cinco rangos de variación: $0-250 \mathrm{~m}$ (baja), 250, I - $500 \mathrm{~m}$ (moderada), 500, I - $750 \mathrm{~m}$ (alta), 750,1 - 1000 m (muy alta) y > 1000 m (extrema). La pendiente se estimó en grados de la siguiente manera: $0^{\circ}-3^{\circ}$ (Illana o suave), $3,1^{\circ}-10^{\circ}$ (moderada), $10,1^{\circ}-25^{\circ}$ (bastante fuerte), $25, \mathrm{I}^{\circ}-45^{\circ}$ (fuerte) y $>45^{\circ}$ (muy fuerte). En este caso no consideramos necesario evaluar la orientación del movimiento puesto que en términos generales no existen cambios abruptos entre las cotas altitudinales del área de estudio. Los dominios vegetacionales se reclasificaron en función de su nivel de productividad primaria (Buono
2008), y la red hídrica se diferenció de acuerdo a la duración de los cuerpos de agua en permanentes, no permanentes y semi-permanentes, según lo indicado por las cartas topográficas del Instituto Geográfico Nacional (IGN) y por nuestras observaciones hechas en el terreno. A esta capa de información se sumaron áreas buffer de $200 \mathrm{~m}$ de distancia a las márgenes de los cuerpos de agua, consideradas como los sectores más óptimos para el desplazamiento debido a los requerimientos del caballo. En relación con esto, fue indispensable otorgarle un cierto valor a cada cuerpo de agua para que su pendiente o cause no actuara como ruta más idónea que un área buffer. No obstante, es probable que en general los cuerpos de agua no actuaran como una barrera infranqueable (e.g. Boschín y Nacuzzi 1979; Musters 2005 [19II]).

De acuerdo con las hojas geológicas también se mapearon los sectores con afloramientos rocosos. A ellos se les otorgó un valor máximo de 100 para que sean identificados como espacios no transitables. Por último, se empleó la información gráfica referida a la caída de nieve y su efecto sobre la mortandad animal durante el invierno (Borrelli et al. 1997 en Stuzenbaum y Oliva 200I). Incluso también la altura y la orientación del terreno permiten ajustar cuestiones relacionadas al riesgo invernal, y a la intensidad de los vientos del oeste y sudoeste. Sobre esta base se pudo evaluar el efecto de las condiciones invernales como factor que inhibe el uso de ciertos espacios, así como su relación con las rutas óptimas.

Para el trazado de las potenciales vías de circulación se conectaron los puntos extremos de la distribución de sitios históricos con presencia indígena, considerándolos dentro de un mismo lapso sin importar una posible falta de contemporaneidad entre ellos (Burmeister 1892 [1891]; Fernández Garay 1994; Lista 1999 [1879]; Moreno 2007 [1877]; Moyano 193 I; Musters 2005 [ I9I I]; Rey Balmaceda 1960; Viedma 1972 [1780-I783]). La localización de estos sitios no hace referencia a una posición exacta, sino que es aproximada, de acuerdo con la información que otorgan los documentos históricos y/o fuentes orales. Con estos puntos se realizó un cálculo isotrópico de rutas óptimas a partir de un mapa de fricción y un mapa de distancia. Este cálculo tiene en cuenta tanto la accesibilidad de los sitios en función de la distancia real entre ellos, como 
el costo que implica atravesar cada sector del terreno para conectarlos. El módulo empleado fue el Shortest Past del Spatial Analysis. Este módulo -una vez estimado el valor acumulado de las diferentes celdas del modelado espacial- selecciona la ruta de menor esfuerzo entre un punto de origen y un punto de destino. También la superposición de las distintas rutas es útil para estimar una posible jerarquización del espacio dentro de los circuitos de movilidad ecuestre. Es decir, una mayor superposición espacial de rutas hace esperable una importancia superior de ese espacio dentro de la circulación ecuestre, antes que otros lugares que han sido menos seleccionados por el cálculo.

\section{RESULTADOS}

A continuación se presentan las rutas óptimas -simbolizadas con líneas negras- generadas a partir del modelo (Figura 2). En varios de sus tramos, las rutas óptimas coinciden con los principales ríos del área. Esto se observa de forma más directa en el trazado de rutas que conectan Isla Pavón con los restantes sitios ubicados en los extremos del área. Los valles de los ríos Chico, Chalía y Santa Cruz son los que representan la mayor parte de los caminos. Sin embargo, también se han generado tramos por espacios internos, como el que une Isla Pavón con el río Chico. Este tramo es de aproximadamente $40 \mathrm{~km}$ de largo y atraviesa áreas buffers próximas entre sí (con distancias menores a $4 \mathrm{~km}$ ), lo que podría haber favorecido su selección.

En otros casos, ciertos tramos de las rutas atraviesan sectores intermedios y alejados de los ríos principales. Por ejemplo, la ruta con origen en Floridablanca atraviesa zonas altas hacia el noroeste, alcanza la cuenca del río Chico recién a la altura de Chunke Aike y luego asciende por el mismo río hasta el paradero Golf. Es interesante mencionar que este trazado intercepta otros sitios históricos a la orilla del río Chico, tales como los mencionados por Musters. En dirección sudoeste, otra ruta con origen en Floridablanca transita las zonas altas que bordean el Bajo de San Julián para acceder a espacios ubicados próximos al lago Viedma. Desde allí alcanza la confluencia de los ríos Chico y Chalía, siendo este último tramo el que marca el camino hasta Orr Aiken. En la conexión entre Floridablanca y Carr Aiken, sobresale el cañadón de Los Paisanos como sector de paso que corre en dirección noreste-sudoeste.

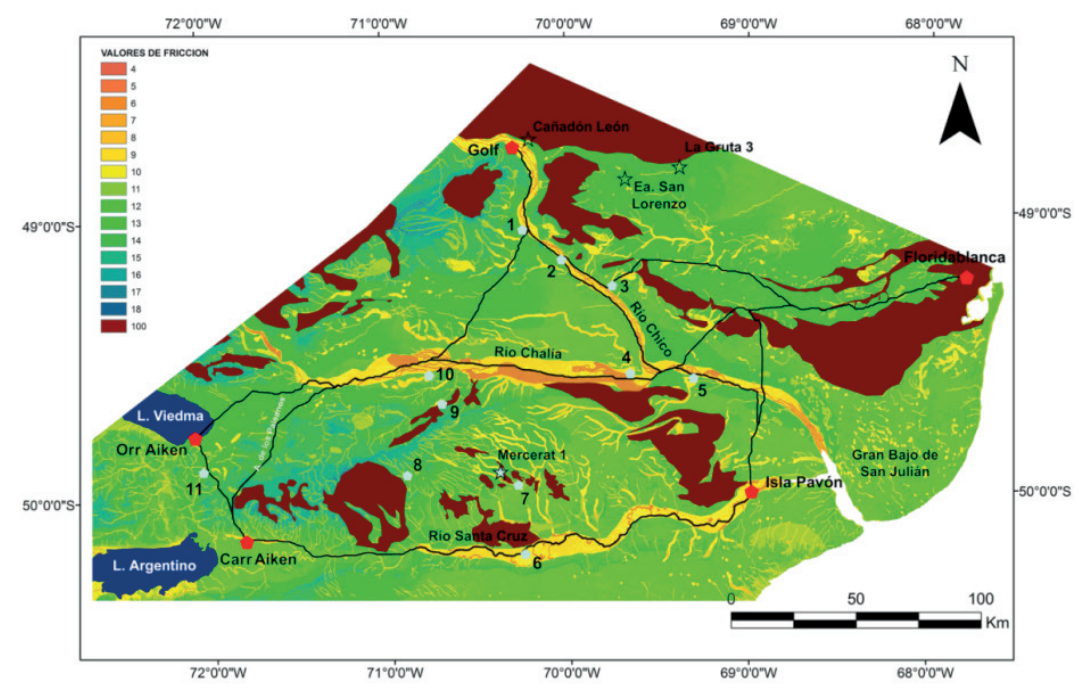

Figura 2: Caminos óptimos entre sitios históricos ubicados en los extremos del área (color rojo). También se señalan la presencia de sitios arqueológicos (estrellas) y otros sitios históricos en colores claros (1- Cerro Ventana, 2-Tayén, 3- Chunke Aike, 4- Cayik, 5- Corpen Aiken (Frequent Winter Quarters of Tehuelches), 6- La Barrancosa, 7- Yaten Guajen, 8- El Cordero, 9- Campamento 1, 10- Shehuen Aiken, 11Paradero Indio).

Figure 2: Optimal pathways between historical sites located at the extreme points of the area (red colour). Archaeological sites (stars) and other historical sites are also highlighted in light colours (1-Cerro Ventana, 2-Tayén, 3-Chunke Aike, 4- Cayik, 5- Corpen Aiken (Frequent Winter

Quarters of Tehuelches), 6- La Barrancosa, 7- Yaten Guajen, 8- El Cordero, 9- Campamento 1, 10- Shehuen Aiken, 11- Paradero Indio). 
Este tramo bordea las pampas altas, evitando los afloramientos rocosos. Finalmente, una ruta interna y el cruce de la cuenca inferior del río Chico sirven de conexión entre Floridablanca e Isla Pavón.

Las rutas con origen en Carr Aiken son las que presentan la mayor cantidad de tramos alejados de los principales ríos. A pesar de que el río Santa Cruz sobresale en la conexión con Isla Pavón, y el Chalía con Corpen Aiken o Chunke Aike, otros tramos con acceso a los sitios cercanos al río Chico (e.g. Shehuen Aike) contemplan el paso por sectores de cañadones verticales. Entre ellos, el cañadón de Los Paisanos es una vía óptima que vincula los ríos Santa Cruz y Chico, permitiendo una conexión directa entre sectores al este del lago Argentino y la cuenca media del río Chico. Por su parte, las zonas cercanas a la margen este del lagoViedma pierden importancia dentro de este trazado de rutas. Siguiendo este modelo, los alrededores del lago Viedma pudieron estar poco vinculados o no ser lugares de tránsito preferenciales para los grupos indígenas históricos. La información histórica sobre el uso estacional de estos espacios y su inaccesibilidad causada por el derretimiento primaveral de la nieve refuerza esta idea (Viedma 1972 [I780-I783]). También se destaca el tramo de ca. $69 \mathrm{~km}$ en línea recta que une
Shehuen Aike con Cerro Ventana, el cual es parte de la ruta interna que atraviesa pequeñas áreas buffer distribuidas cada 4 a $8 \mathrm{~km}$. Dentro de la lógica de la jerarquización espacial, también existen zonas en las que se superponen rutas óptimas. En este esquema de zonas preferenciales para la circulación ecuestre predominan los principales valles fluviales, incluyendo también posibles traslados por cañadones de tendencia vertical; $y$ para los sectores bajos con presencia aislada de fuentes de agua, o bien a más de $10 \mathrm{~km}$ entre sí, se estima un bajo nivel de uso.

A esto se suma la disponibilidad estacional vs. anual de los espacios (Figura 3), a partir de la cual se puede trazar una línea recta imaginaria que une Cañadón León I con Isla Pavón (Figura 2). Esta línea imaginaria da cuenta de la disponibilidad diferencial de espacios durante el invierno, siendo los sectores de valles fluviales, las zonas bajas circundantes y los espacios ubicados hacia el este de ella, zonas con mayor disponibilidad anual. Los sectores internos más elevados y entre valles localizados al oeste del área, debieron ser más estacionales. Tales condiciones de estrés invernal pudieron ser más severas durante la Pequeña Edad de Hielo -ca. 1350 - I850 DC -. En el área de estudio las más altas precipitaciones con respecto a la actualidad (Meyer y Wagner

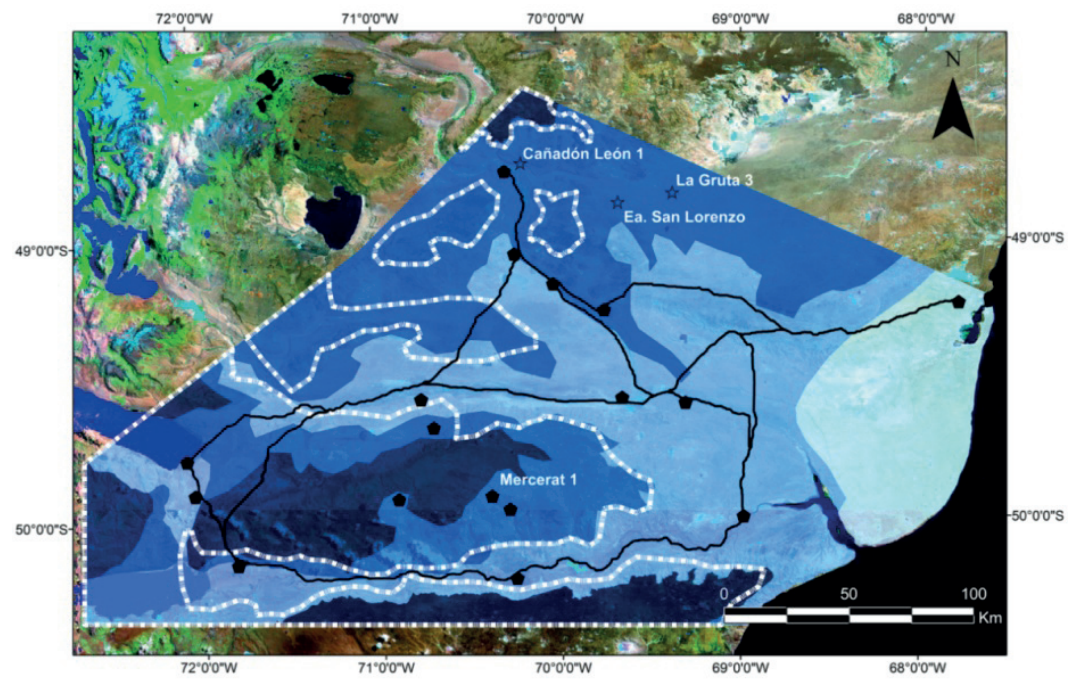

Figura 3: Se presenta el modelo de circulación óptima y la localización de sitios arqueológicos en relación con el riesgo invernal, cuyos valores aumentan de sectores muy claros (Gran Bajo de San Julián) a muy oscuros (las zonas más occidentales y aquellas por encima de los $400 \mathrm{msnm}$ ). También se señalan los sectores con cobertura nival actual durante el invierno (polígonos con línea punteada blanca).

Figure 3: The optimal movement model and the place of archaeological sites in regard to the winter risk factor, whose values increase from very clear sectors (Gran Bajo de San Julián) to very dark (the westernmost areas and those above $400 \mathrm{~m}$ ) is presented. Current sectors with snow cover during the winter season (polygons with white dotted line) are also reported. 
2009) debieron generar condiciones climáticas que incluyeran niveles de evaporación más bajos, menores temperaturas frías anuales, temporadas de crecimiento acortadas, heladas más frecuentes y líneas de nieve más bajas. Es posible que todos estos fenómenos asociados al invierno implicaran un aumento de los costos y de los tiempos de traslado, dado que ciertos caminos internos que se desempeñan como rutas óptimas debieron quedar inhabilitados temporalmente. Un ejemplo de esto serían aquellos pasos internos que comunican con la zona de los lagos pericordilleranos. En tales casos creemos que los principales valles fluviales pudieron funcionar como rutas alternativas $y$ de menor riesgo durante el invierno. Asimismo, los grandes lagos pudieron funcionar como atemperantes del rigor invernal y sus costas -que no suelen congelarse- lograron estar disponibles para ser ocupadas, accediendo a ellas a través de los principales valles.

En el área se han localizado algunos sitios arqueológicos y resulta interesante comparar su ubicación con la prevista por el modelo. Entre éstos, sólo Cañadón León I está próximo a una ruta óptima (Figura 3). Éste fue señalado como un probable sitio histórico correspondiente al siglo $X X$, dado que presenta una concentración discreta de materiales en superficie, incluyendo artefactos líticos, vítreos, fragmentos de vidrio, gres y metal, cuya estricta pennecontemporaneidad no pudo ser demostrada (Cirigliano 2013). Otros dos sitios arqueológicos son Mercerat I, donde se recuperaron instrumentos de vidrio (Cirigliano y Vommaro 2014) y la Gruta 3 , donde se identificó un espécimen de fauna europea con huellas de corte junto con artefactos líticos (Cirigliano y Vommaro 2013). Ambos sitios no responden a las expectativas del modelo de circulación óptima para tiempos ecuestres. Esto mismo ocurre con un raspador de vidrio encontrado de manera aislada en la Ea. San Lorenzo (Vector Argentina S.A. 2005), al sur del Macizo del Deseado. Estos tres sitios se localizan en sectores internos, alejados de las rutas óptimas y en zonas con niveles medios a altos de riesgo invernal (Borrelli et al. 1997 en Sturzenbaum y Borrelli 200I). A continuación discutiremos las implicancias que esto tiene sobre la utilidad del modelo de rutas óptimas para predecir la localización de sitios arqueológicos en el área de estudio.

\section{DISCUSIÓN}

Dada la escasez de sitios de momentos históricos localizados sobre base arqueológica, los parajes señalados por viajeros y exploradores son la principal evidencia para discutir los circuitos de movilidad de los grupos cazadores recolectores ecuestres entre las cuencas de los ríos Chico y Santa Cruz. De todos ellos, los ubicados en los extremos del área sirvieron para modelar las rutas óptimas. Por este motivo, la evaluación del ajuste de la localización de los sitios mencionados para zonas interiores con el modelo de la circulación ecuestre, cobra importancia para establecer el potencial del modelado en la búsqueda de nuevos sitios arqueológicos. Al mismo tiempo, esto otorgaría mayor fiabilidad a los documentos históricos acerca de cuáles eran los espacios que solían ocuparse.

Según el modelo es esperable que se registren -en mayor medida- hallazgos vinculados con rutas óptimas que corren a lo largo de los principales valles fluviales, y con sectores de cotas bajas, escasa pendiente, buenas pasturas y amplia disponibilidad anual. En términos generales, la información histórica presenta un mayor ajuste a estas expectativas que la información arqueológica. En particular, nos referimos a los sitios históricos registrados por las fuentes al interior del área (ver Figura 2), incluyendo otros que aquí no han sido graficados (e.g. CailletBois 1936: 23 en Aguerre 2009:I36-137; Lista 1999 [1879]; Peláez 2000). También la ruta señalada por Viedma desde Bahía San Julián hasta el lago Capar se solapa con una ruta óptima generada (Ministerio de Cultura, Archivos estatales de España 1786). En cambio, los sitios arqueológicos Mercerat I, La Gruta 3 y Ea. San Lorenzo se localizan en sectores de elevación moderada, con niveles medios a altos de riesgo invernal y alejados de las rutas óptimas. Una alternativa que podría explicar su bajo grado de ajuste es su posible vinculación con actividades de carácter principalmente logístico y/o estacional. Por su parte, el sitio Cañadón León I está próximo a una ruta óptima, generando expectativas acerca de una relación más estrecha con los circuitos de movilidad ecuestre. No obstante, su localización en una zona con alto riesgo invernal indicaría un probable uso estacional. Respecto de esto, creemos que las exploraciones futuras vinculadas a las expectativas del modelo servirán para contrastarlo y arrojar luz sobre el uso de espacios asociados a él. 
El modelado también añade fiabilidad a un potencial uso de los cañadones como zonas de traslado habitual, tal como señalan los documentos históricos. Los cañadones, e incluso algunas zonas buffer, son vías que atraviesan sectores internos, estableciendo conexiones entre los principales valles fluviales; y por ende, debieron servir para reducir notablemente las distancias del traslado. De acuerdo con el modelo, los cañadones que corren en dirección norte - sur pudieron ser preferenciales para la circulación humana, dado que también ofrecen reparo de los vientos predominantes. Sin embargo, la mayoría de estos espacios debieron ser de uso estacional, siendo que presentan niveles medios a altos de riesgo invernal, junto con lagunas y cañadones generalmente efímeros (Borrelli et al. 1997 en Sturzenbaum y Borrelli 200 I; Panza et al. 2005).

La naturaleza de la estacionalidad es de suma utilidad parar generar expectativas sobre la intensidad diferencial en el uso humano del espacio (e.g. Gradin 1976; Goñi 2000; Pallo 20I2), y especialmente entre los grupos ecuestre, cuya movilidad debió estar basada principalmente en dar respuesta a las necesidades de forraje y agua para los caballos (Osborn 1983; Zeldeño et al. 2014). Entre las poblaciones ecuestres de Norteamérica, los inviernos severos y la duración media de la estación de crecimiento de las plantas han sido vistos como limitantes ambientales previsibles y observables en la búsqueda de forraje para los caballos y su reproducción (Osborn 1983). Los grupos ecuestres movían frecuentemente sus campamentos a corta distancia por los rápidos descensos de forraje acaecidos poco después de ocupar un campamento (Kelly 1995). Situaciones de este tipo pudieron haber ocurrido en el área de estudio (Cirigliano 2016). Una menor intensidad de ocupación por estrés invernal es probable que se vincule con sectores hacia el oeste del área, incluyendo espacios por encima de los 400 msnm, en los que son más probables la falta de forraje y los eventos de mortandad animal por caída de nieve (Borrelli et al. 1997 en Sturzenbaum y Borrelli 200I). En términos de movilidad, el modelo pone de relieve que los sectores hacia el este y por debajo de los $250 \mathrm{msnm}$, pudieron proporcionar mayores opciones de carácter residencial durante el invierno, y también en otras épocas del año. En este caso, la movilidad logística pudo haber sido la alternativa más conveniente para atender las necesidades de los caballos durante el invierno (Osborn 1983), lo que ha sido señalado como un proceso de extensificación sensu Binford (200I). Entre las opciones de paso los cañadones y el congelamiento de algunos sectores de los principales ríos pudieron ser buenas alternativas para la circulación y el cruce de los valles fluviales (Musters 2005 [19II]). Contrariamente a esto, el aumento de los caudales de los ríos a causa de los deshielos primaverales debió imponer un limitante a la circulación ecuestre (Viedma 1972 [1780-1783]).

En la búsqueda de nuevos sitios históricos, la expectativa es que los lugares con mayor disponibilidad anual hayan sostenido una mayor intensidad de uso, y en consecuencia una mayor frecuencia promedio de artefactos que otros posibles sitios estacionales $\circ$ al paso, como los registrados en la porción central del área (Cirigliano 2016). También otras fuentes de datos -hacia el oeste y el este del área- refuerzan esta idea. Por un lado, la información arqueológica en cotas altas de la margen norte del lago Argentino, dado que allí se registran actividades vinculadas con fines específicos, como el procesamiento de chulengos (Franco y Borrero 1995). Por otro lado, los documentos históricos que refieren a Corpen Aike (Frequent Winter Quarters of Tehuelches) y al campamento próximo a Floridablanca como sitios de ocupación prolongada (Buscaglia 2012; Musters 2005 [191 I]; Nacuzzi I99I, 2005;Viedma 1972 [1780-1783]).

En términos generales, el modelo de las potenciales vías de circulación cubre la mayor parte de las expectativas asociadas a la búsqueda de sitios arqueológicos de momentos ecuestres. Esto se debe a que ha sido aplicado a escala regional y al período histórico en general, sobre la base de que la mayoría de los lugares para acampar en Patagonia meridional eran postas durante los traslados (Boschín y Nacuzzi 1979). No obstante esto, existen algunas cuestiones que quedan fuera del modelo. Entre ellas, es claro que el uso de la escala regional influye en las observaciones realizadas, por lo que aplicar una escala local podría ser una puerta de entrada a considerar nuevas rutas óptimas (e.g. el cañadón que intercepta el sitio arqueológico Mercerat I). También en la escala temporal existen datos que podrían llevarnos a considerar sectores que escapen al modelo propuesto. En particular nos referimos a ciertos lapsos del siglo $X X$ en los que algunos 
grupos ecuestres pudieron replegarse a sectores internos y usualmente no transitados, como forma de evitar el contacto ante el avance de criollos o criollo-europeos (Aguerre 2000; ver también Goñi y Nuevo Delaunay 2009 para casos de ocupaciones alternativas a las reservas indígenas). Esto pudo dar lugar a que zonas no óptimas fueran especialmente seleccionadas por los grupos indígenas para ser ocupadas. Adicionalmente, se debe contemplar la atracción que pudieron haber generado las estancias como proveedoras de materias primas exóticas y de trabajo remunerado durante el avance del poblamiento criollo-europeo en el área de estudio (Cirigliano 2016). Extender el análisis espacial y la búsqueda arqueológica a este tipo de situaciones particulares servirían de complemento al modelo aquí realizado. $A$ esto se suman otros factores que afectan la contrastación del modelo. Entre ellos, la preservación y la visibilidad diferencial del registro arqueológico que operan a favor de los sitios programados antes que de los sitios al paso (Borrero et al. 2008). También se añade la escasez de artefactos diagnósticos -como aquellos manufacturados con elementos exóticos- en los sitios arqueológicos de momentos históricos, lo que explica en buena medida la dificultad para identificarlos (Cirigliano 2016).

\section{CONCLUSIÓN}

Buena parte de la información mencionada por viajeros y exploradores acerca de los parajes indígenas y las vías de paso ha quedado reflejada en el modelo de circulación óptima. Esto da mayor fiabilidad a las fuentes históricas empleadas y por otro lado, otorga un buen grado de predictibilidad al modelo. En cambio, la localización de los escasos sitios arqueológicos no presentó el mismo grado de ajuste al modelo, lo que podría explicarse en función de la movilidad logística y/o estacional. También existen otra clase de factores que exceden la movilidad óptima, y por ende nuestro modelo, como la existencia de vecinos, la búsqueda de intercambio, entre otros (e.g. Kelly 1995).

El uso de SIG nos ha ofrecido una solución metodológica para comenzar a zanjar cuestiones vinculadas con la escasez actual del registro arqueológico de momentos históricos y la potencial localización de sitios asociados con la movilidad indígena ecuestre. En este sentido, nuestro modelo es predictivo y constituye una herramienta para diseñar trabajos de campo a futuro, reorientando la búsqueda arqueológica hacia sectores aún no prospectados y que posiblemente estén vinculados con ocupaciones de carácter más prolongado. Asimismo, ha resultado útil para discutir cuestiones vinculadas con la intensidad de uso del espacio y la estacionalidad. Siguiendo la propuesta del modelo, los espacios hacia el este del área podrían registrar ocupaciones de carácter más prolongado y por lo tanto, materialmente más visible. Los futuros trabajos de campo permitirán contrastar las expectativas del modelo, y generar nuevas observaciones para aproximarnos a mayores conclusiones sobre la movilidad de los grupos ecuestres en Patagonia Meridional.

Agradecimientos: A Nora Franco y Luis Borrero por su apoyo, colaboración y comentarios. Este trabajo fue financiado por los proyectos PIP 0356 (CONICET) y UBACyT 0I/W404,y se enmarca dentro de nuestras becas CONICET (de finalización de doctorado y postdoctoral). A los evaluadores por sus valiosos comentarios.

\section{NOTAS}

I Nacuzzi (2005) estudia el lapso I770-I870 y señala que no se puede generalizar los movimientos durante la totalidad del período histórico (I520-1950 años DC) dado el rápido proceso de aculturación vivenciado por los grupos indígenas. En este trabajo abarcamos hasta primera mitad del siglo $X X$ debido a que el avance de la frontera ovino ganadera en nuestra zona se produce entre 1900 y 1970 , dependiendo de las propiedades de distintos sectores (Barbería 1995).

\section{BIBLIOGRAFÍA}

Aguerre, A. M. 2000. Las Vidas de Pati en la Toldería Tehuelche del Río Pinturas y el después. Facultad de Filosofía y Letras, Universidad de Buenos Aires, Buenos Aires.

----2009. Genealogía de familias tehuelches-araucanas de la Patagonia central y meridional argentina. Editorial de la Facultad de Filosofía y Letras, Universidad de Buenos Aires, Buenos Aires.

Barbería, E. M. 1995. Los Dueños de la Tierra en la Patagonia Austral, 1880-1920. UNPA, Río Gallegos.

Binford, L. R. 1980. "Willow smoke and dog 's tails: hunter-gatherer settlement systems and archaeological site formation". American Antiquity 45 (I): 4-20. 
----200I. Constructing Frames of Reference. An Analytical Method for Archaeological Theory Building Using Ethnographic and Environmental Data Sets, editado por Blumenschine, R. J. y M. M. Selvaggio. University of California Press. California.

Borrero, L. A. 2005. "The archaeology of the Patagonian Deserts: Hunter-Gatherers in a cold desert”. En Desert Peoples. Archaeological Perspectives, editado por P. Veth, M. Smith y P. Hiscock, Pp. I42-I 58. Oxford, Blackwell.

Borrero, L. A. y F. Carballo Marina. 1998. "Proyecto Magallania: La cuenca superior del tío Santa Cruz”. En Arqueología de la Patagonia Meridional (Proyecto Magallania), compilado por L. A. Borrero, Pp. II-27. Ediciones Búsqueda de Ayllu, Concepción del Uruguay.

Borrero, L. A. y N. V. Franco. 2000."Cuenca superior del río Santa Cruz: perspectivas temporales”. En Desde el País de los Gigantes. Perspectivas Arqueológicas en Patagonia, tomo II, Pp. 345356. Universidad Nacional de la Patagonia Austral, Río Gallegos.

Borrero, L. A., J. Charlin, R. Barberena, F. M. Martin, K. Borrazzo, L. L'Heureux. 2008. Circulación humana y modos de interacción al sur del río Santa Cruz. En Arqueología del Extremo Sur del Continente Americano. Resultados de Nuevos Proyectos, compilado por L. A. Borrero y N. V. Franco, Pp. I55-I 74. IMHICIHU - CONICET, Buenos Aires.

Boschín, M. T. y L. R. Nacuzzi. 1979. Ensayo Metodológico para la Reconstrucción Etnohistórica. Su Aplicación a la Comprensión del ModeloTehuelche Meridional. Colegio de Graduados en Antropología. Serie Monográfica $N^{\circ} 4$, Buenos Aires.

Buono, G. 2008. "La oferta de forraje de los pastizales naturales”. En Manejo de Pastizales “Evaluación Predial”, editado por INTA-Trelew, Pp. 9- I3. http://organismos.chubut.gov.ar

Burmeister, C. V. 1892 [1891]. "Nuevos datos sobre el Territorio Patagónico de Santa Cruz". Revista del Museo de La Plata IV: 22I-256.

Buscaglia, S. 2012. Poder y Dinámica Interétnica en la Colonia Española Floridablanca. Una Perspectiva Histórica y Arqueológica (Patagonia, Argentina, Siglo XIII). Editorial Académica Española, Buenos Aires.

Carballo, M., F., L. A. Borrero, N. V. Franco, J. B. Belardi, V. D. Horwitz, A. S. Muñoz, P. Campan, F. M. Martín, F. Borella, M. F. García, F. Muñoz, F. Savanti y J. L. Lanata. 1999. "Arqueología de la costa del lago Argentino, río La Leona y pampas altas intermedias (Provincia de Santa Cruz, Argentina)". Praehistoria 3:13-33.

Cirigliano, N. A. 2013. "Primera aproximación al sitio Cañadón León I. Los Materiales de superficie (Provincia de Santa Cruz, Argentina)". En Tendencias Teórico - Metodológicas y Casos de Estudio en la Arqueología de la Patagonia, compilado por F. Zangrando, R. Barberena, A. F. Gil, G. A. Neme Gustavo, M. A. Giardina, L. Luna, C. Otaola, S. L. Paulides, L. M. Salgan y A. M.Tívoli, Pp. 317-324. SAA, INAPL, Museo de Historia Natural de San Rafael, Buenos Aires.

--- 2016. Movilidad de grupos indígenas y aprovechamiento de materias primas entre el extremo sur del Macizo del Deseado y la cuenca del río Santa Cruz durante los últimos 2.000 años (Provincia de Santa Cruz,Argentina). Tesis para optar al grado de doctor. Facultad de Filosofía y Letras, Universidad de Buenos Aires.

Cirigliano, N. A. y M. N. Vommaro. 20I3."Historical occupations at the Southern Part of the Deseado Massif: The case of La Gruta 3 (Santa Cruz, Argentina)". En Program of the 78 th Annual Meeting, Society for America Archaeology, editado por Society for America Archaeology, Pp I0 I. Honolulu, Hawaii.

---- 20l4. "Un asentamiento histórico en cercanías del cañadón Mercerá (provincia de Santa Cruz, Argentina)". En Entre Pasados y Presentes IV. Estudios Contemporáneos en Ciencias Antropológicas, editado por A. Castro Esnal, M. L. Funes, M. Grosso, N. Cuperszmit, A. Murgo y G. Romero, Pp. 280-296.AINA, Buenos Aires.

Del Castillo, A. 1979 [1887]. Exploración de Santa Cruz y Costas del Pacífico. Ediciones Marymar, Buenos Aires.

Ewers, J. C. 1955. The Horse in Blackfoot Indian Culture:With Comparative Material from OtherWestern Tribes. Bureau of American Ethnology Bulletin No. I59.

Fernández Garay, A. 1994. Testimonios de los Últimos Tehuelches. Textos Originales con Traducción y Notas LingüísticoEtnográficas. Archivo de Lenguas Indoamericanas. Nuestra América. Instituto de Lingüística.

Franco, N. V. 2002. Estrategias de utilización de recursos líticos en la cuenca superior del río Santa Cruz. Tesis doctoral. Facultad de Filosofía y Letras, Universidad de Buenos Aires.

--- 2008. "La estructura tecnológica regional y la comprensión de la movilidad humana: tendencias para la cuenca del río Santa Cruz". En Arqueología del Extremo Sur del Continente Americano. Resultados de Nuevos Proyectos, compilado por L. A. Borrero y N. V. Franco, Pp. II9-I54. IMHICIHU - CONICET, Buenos Aires

Franco, N. V. y L. A. Borrero. 1995."Bifaces, Guanacos and Other Resources. The Evolution of Patagonian Populations". Actas del 60vo. Annual Meeting, Society for American Archaeology, Minneapolis.

Franco, N. V., M. Cardillo, C. Otaola, N. Arregui y E. Gáal. 2007a. "Tendencias preliminares en el registro arqueológico del curso medio y superior de arroyo El Lechuza, provincia de Santa Cruz, Argentina". Intersecciones en Antropología 8:27I-285.

Franco, N. V., C. Otaola y M. Cardillo. 2007b. "Resultados de los trabajos exploratorios realizados en la margen norte del río Santa Cruz (provincia de Santa Cruz, Argentina)". En Arqueología de Fuego-Patagonia. Levantando Piedras, Desenterrando Huesos... y Develando Arcanos, editado por F. Morello, M. Martinic, A. Prieto y G. Bahamonde, pp. 54I-553. Ediciones CEQUA, Punta Arenas.

Franco, N.V., M Martucci, P. Ambrústolo, G Brook, M.V. Mancini y N. Cirigliano. 20I0. “Ocupaciones humanas correspondientes a la transición Pleistoceno-Holoceno al sur del Macizo del Deseado: El área de La Gruta (Provincia de Santa Cruz, Argentina)". Relaciones de la Sociedad Argentina de Antropología XXXV: 30I-308.

Franco, N. V., P. Ambrústolo, A. Acevedo, N. Cirigliano y M. Vommaro. 20I3a. "Prospecciones en el sur del Macizo del Deseado. Los casos de La Gruta y Viuda Quenzana”. En Tendencias Teórico - Metodológicas y Casos de Estudio en la Arqueología de la Patagonia, compilado por F. Zangrando, R. Barberena, A. F. Gil, G.A. Neme Gustavo, M.A. Giardina, L. Luna, C. Otaola, S. L. Paulides, L. M. Salgan y A. M. Tívoli, Pp. 37I-378. SAA, INAPL y el Museo de Historia Natural de San Rafael, Buenos Aires.

Franco, N. V., N. Cirigliano, D. Fiore, M. Ocampo 
y A. Acevedo. 20I3b. "Las ocupaciones del Holoceno tardío en los cañadones basálticos del norte del río Santa Cruz (Patagonia, Argentina)". Intersecciones en Antropología (en prensa).

Furque, G. 1973. “Descripción geológica de la Hoja 58b Lago Argentino”. Boletín del Servicio Nacional Minero y Geológico 140: I-49.

Goñi, R. A. 1988. "Arqueología de momentos tardíos en el Parque Nacional Perito Moreno (Santa Cruz, Argentina)". Precirculados del IX Congreso Nacional de Arqueología Argentina, pp. |40-I5 I. Buenos Aires.

---- 2000. “Arqueología de momentos históricos fuera de los centros de conquista y colonización: un análisis de caso en el sur de la Patagonia". En Desde el País de los Gigantes. Perspectivas arqueológicas en Patagonia. Tomo I, editado por J. B. Belardi, F. Carballo Marina y S. Espinosa, Pp. 283-293.UARG-UNPA, Río Gallegos.

---- 2013. "Reacomodamientos poblacionales de momentos de momentos históricos en el noroeste de Santa Cruz. Proyecciones arqueológicas”. En Tendencias Teórico - Metodológicas y Casos de Estudio en la Arqueología de la Patagonia, compilado por F. Zangrando, R. Barberena, A. F. Gil, G. A. Neme Gustavo, M. A. Giardina, L. Luna, C. Otaola, S. L. Paulides, L. M. Salgan y A. M.Tívoli, Pp. 389-396. SAA, INAPL y el Museo de Historia Natural de San Rafael, Buenos Aires.

Goñi, R. y A. Nuevo Delaunay. 2009.“La Arqueología como "fuente" de la Historia". En Arqueología de la Patagonia. Una Mirada desde el Último Confin, editado por M. Salemme, F. Santiago, M. Álvarez, E. Piana, M. Vázquez y E. Mansur, Tomo I, Pp. I49-I 58. Utopías, Ushuaia.

Gradin, C. J. 1976. "Parapetos de piedra y grabados rupestres de la meseta del lago Buenos Aires". Revista del Museo de Historia Natural de San Rafael II( I/4): 315/337 Buenos Aires

---- 2000. Más allá y más acá del río Santa Cruz. Nuevo Offset.

Halvorsen, P. 2009. La Leona: Historia de Balsas, Boliches y Enredos. Patagonia Sur Libros, Buenos Aires.

---- 20I I. Identidades Enmascaradas en la Patagonia. Uniones entre Nativos y Foráneos. Patagonia Sur, Buenos Aires.

Kelekna, P. 2009. The Politico-Econimic Impact of the Horse on the OldWord Cultures.An Overview, editado porV.H. Mair, pp I-3I. SINO-Platonic papers, Número 190, University of Pennsylvania, Filadelfia.

Kelly, R. L. 1995. The Foraging Spectrum. Diversity in HunterGatherer Lifeways. Smithsonian Institution Press, Washington DC.

Lista, R. 1999 [1879]. Viaje a la Patagonia Austral (Complemento del Viaje al País de los Tehuelches). Confluencia, Buenos Aires.

Mancini, M. V., N. Franco, and G. Brook. 2012. "Early human occu-pation and environment south of the Deseado massif and south of Lago Argentino (Argentina)". En Southbound: Late Pleistocene peopling of Latin America, editado por L. Miotti, M. Salemme, N. Flegenheimer y T. Goebel, Pp. 197-200. Center for the Study of the First Americans, A\&M University, College Station. Texas

Martinic, M. 1984. "San Gregorio, centro tehuelche meridional". Anales del Instituto de la Patagonia I5:I I-25.

---- 1995. Los Aónikenk, Historia y Cultura. Ediciones Universidad de Magallanes, Punta Arenas.
Matteucci, S. D. y V. Scheinsohn. 2004. "Procesamiento de imágenes, SIG y modelos ecológicos aplicados a la arqueología”. Geofocus 4: 93-109.

Meyer, I. y S. Wagner. 2009. "The little Ice Age in Southern South America: Proxy and model based evidence". En Past Climate Variability in South America and Surrounding Regions: From the Last Glacial Maximum to the Holocene, editado por F.Vimeux, F. Sylvestre y M. Khodri, Pp. 395- 4I2. Springer, Netherlands.

Ministerio de Cultura, Archivos estatales de España. 1786. Mapa del Marqués de Loreto http://bahiasinfondo. Blogspot .com.arl 2012/02 /buscando-mapas-viejos-de-la-patagonia. html

Moreno, F. P. 2007 [1877]. Exploración de la Patagonia sur II. El lago Argentino y los Andes meridionales (1877). Ediciones Continente, Buenos Aires.

Moreno, J. M. 2008. Arqueología y Etnohistoria de la Costa Patagónica Central en el Holoceno Tardío. Secretaría de Cultura de Chubut.

Moyano, C. M. 1881 . A través de la Patagonia. Informe de Viaje y Exploración desde Santa Cruz al Chubut. Imprenta de La Tribuna Nacional, 86 -San Martín-86, Buenos Aires.

---- 193I Viajes de exploración a la Patagonia (1877-1890). Imprenta Mercatali, Buenos Aires.

Musters, G. C. 2005 [I9II] Vida entre los Patagones. El Elefante Blanco, Buenos Aires

Nacuzzi, L. R. 1991. "La cuestión del nomadismo entre los tehuelches. Cuadernos de Etnohistoria”. Memoria Americana I: $103-134$.

---- 2005. Identidades Impuestas. Tehuelches, Aucas y Pampas en el Norte de la Patagonia. Sociedad Argentina de Antropología (2 edición), Buenos Aires.

Nacuzzi, L. R. y C. Pérez de Micou. 1994. "Rutas indígenas y obtención de recursos económicos en Patagonia”. Memoria Americana 3: 91-104.

Narborough, J. 2007[1694]. "El viaje de Sir John Narbrough al Mar del Sur". En Un relato de diversos viajes y descubrimientos recientes, editado por R. C. Paredes, PP 73-I65. Colección Reservada del Museo del fin del mundo. Eudeba, Buenos Aires.

Oliva, G., L. González, P. Rial y E. Livrachi. 200I. “Áreas Ecológicas de Santa Cruz y Tierra del Fuego". En Ganadería ovina sustentable en la Patagonia Austral. Tecnología de manejo extensivo, editado por P. Borrelli y G. Oliva, Pp. 4I- 82. INTA, Río Gallegos.

Osborn, A. 1983. "Ecological Aspects of Equestrian Adaptations in Aboriginal North America". American Anthropologist 85 (3): 563-59l.

Panza, J. L., L. E. Sacomani y J. C. Cobos. 2005. Hoja Geológica 4969-III Laguna Grande. Programa Nacional de Cartas Geológicas de la República Argentina I:250.000. Provincia de Santa Cruz. Boletín N³62. Instituto de Geología y Recursos Minerales y SEGEMAR, Buenos Aires.

Pallo, M. C. 2012."El estrés invernal como generador de áreas marginales en Patagonia meridional durante el Holoceno tardío”. Comechingonia Virtual VI (I):86-I I 4.

Peláez, P. A. 2000. Nueva información referida a una ruta tehuelche del siglo XIX. En Desde el país de los gigantes. Perspectivas 
arqueológicas en Patagonia. Tomo I, editado por J. B. Belardi, F. Carballo Marina y S. Espinosa, tomo I, pp. 283-296. UARG-UNPA, Río Gallegos.

Pigafetta, A. 200I [1899]. PrimerViaje alrededor del Mundo. El Elefante Blanco (I ${ }^{\text {a }}$ edición), Buenos Aires.

Priegue, C. N. 2007. En Memoria de los Abuelos. Historia de Vida de Luisa Pascual, Tehuelche. Publitek, Bahía Blanca.

Raedeke, L. D. 1979. Population dynamics and socioecology of the guanaco (Lama guanicoe) of Magallanes, Chile. Tesis de doctorado inédita, University of Washington, Seattle.

Rey Balmaceda, R. C. 1960. Geografia histórica de Patagonia: 1870. Tesis para optar al grado de doctor. Facultad de Filosofía y Letras, Universidad de Buenos Aires.

Soto, J. 2000. "Satélites Meteorológicos", En El Gran Libro de la Provincia de Santa Cruz. Patagonia-Argentina. Tomo I, editado por A. García y E Mazzoni, PP. I0I-II5. Madrid, ALFA Centro Literario y Milenio ediciones, Madrid.

Sturzenbaum, P. y P. Borrelli. 200I. "Manejo de riesgos climáticos". En Ganadería Ovina Sustentable en la Patagonia Austral. Tecnologías de Manejo Extensivo, editado por P. Borreli y G. Oliva, Pp. 255-270. INTA- Santa cruz. Editorial ErreGé \&Asociados, Buenos Aires.

Stine, S. 1994. "Extreme and persistent drought in California and Patagonia during medieval time”. Nature 369: 546549.

Vector Argentina S. A. 2005. "Estudios de la línea de base del proyecto Manantial. Informe arqueológico". NWH. Informe de Impacto Ambiental Proyecto Manantial Espejo. Copias disponibles en Gobernador Gregores, Santa Cruz. Manuscrito en posesión de los autores.

Viedma, A. 1972 [1780-1783]. "Diario de Viedma”. En Colección Pedro De Angelis, Tomo VIII, volumen B, pp. 845-936. Plus Ultra, Buenos Aires.

Wissler, C. 1914 "The Influence of the Horse in the
Development of Plains Culture". American Anthropologist 16 (I): $\mathrm{I}-25$.

Zerdeño, M. N., J.A. Ballenger y J. R. Murray. 2014. "Landscape Engineering and Organizational Complexity among Late Prehistoric Bison Hunters of the Northwestern Plains". Current Anthropology 55(I): 23-58. 